\title{
Entropy and kinetics of point defects in two-dimensional dipolar crystals
}

\author{
Wolfgang Lechner,,${ }^{1,2, *}$ David Polster, ${ }^{3}$ Georg Maret,${ }^{3}$ Christoph Dellago, ${ }^{4}$ and Peter Keim ${ }^{3}$ \\ ${ }^{1}$ Institute for Quantum Optics and Quantum Information, Austrian Academy of Sciences, 6020 Innsbruck, Austria \\ ${ }^{2}$ Institute for Theoretical Physics, University of Innsbruck, 6020 Innsbruck, Austria \\ ${ }^{3}$ Department of Physics, University of Konstanz, D-78457 Konstanz, Germany \\ ${ }^{4}$ Faculty of Physics, University of Vienna, Boltzmanngasse 5, 1090 Vienna, Austria
}

(Received 16 September 2014; revised manuscript received 15 January 2015; published 5 March 2015)

\begin{abstract}
We study in experiment and with computer simulation the free energy and the kinetics of vacancy and interstitial defects in two-dimensional dipolar crystals. The defects appear in different local topologies, which we characterize by their point group symmetry; $C_{n}$ is the $n$-fold cyclic group and $D_{n}$ is the dihedral group, including reflections. The frequency of different local topologies is not determined by their almost degenerate energies but is dominated by entropy for symmetric configurations. The kinetics of the defects is fully reproduced by a master equation in a multistate Markov model. In this model, the system is described by the state of the defect and the time evolution is given by transitions occurring with particular rates. These transition rate constants are extracted from experiments and simulations using an optimization procedure. The good agreement between experiment, simulation, and master equation thus provides evidence for the accuracy of the model.
\end{abstract}

DOI: 10.1103/PhysRevE.91.032304

PACS number(s): 82.70.Dd, 61.72.-y

\section{INTRODUCTION}

The microscopic dynamics and interaction of defects, such as dislocations, vacancies, and interstitials, are key to a variety of macroscopic phenomena of materials [1,2]. In two-dimensional (2D) systems [3-7], the peculiar two-step melting is a result of dislocation and disclination interactions, and melting is mediated by the formation and subsequent dissociation of dislocation pairs [8-15]. Dislocations pairs in $2 \mathrm{D}$ crystals also form as the result of spontaneous clustering of interstitials and vacancies introduced into the systems [16]. The dynamics and interaction of interstitials and vacancies is even related to exotic phases such as supersolidity $[17,18]$. Nevertheless, even though individual defects and their displacement fields are well described by elasticity theory, the precise kinetics and the sign of the interaction cannot be captured by the theory $[19,20]$. The open question is how the defect kinetics emerges from the nonlinear effects near the defect centers. Video microscopy of two-dimensional colloidal crystals together with optical tweezers now allow us to investigate such nonlinear effects with single-particle resolution in real time [21-25].

Here, we show that the dynamics of defects in twodimensional dipolar crystals can be fully described by a sequence of jumps between states that are defined by the local displacements in the vicinity of the defect centers. The equilibrium probabilities of the states (i.e., the populations) are a result of the interplay between entropic and energetic contributions. We find that the different contributions can be understood quantitatively from statistical mechanics by a harmonic expansion of the energy around the minima corresponding to the various defect states. The kinetics of the defects follows a master equation for which we measure the transition matrix from a long experimental trajectory with the aid of an optimization routine. The results from experiments are compared with the results from Monte Carlo

\footnotetext{
*w.lechner@uibk.ac.at
}

simulations. The equilibrium probabilities from simulation and experiment are in qualitatively good agreement but show systematic differences for stiff crystals.

\section{EXPERIMENTAL SETUP AND MODEL}

We consider a system of superparamagnetic colloidal particles $(4.5 \mu \mathrm{m}$ in diameter) confined by gravity to two dimensions on a flat interface. Two situations were realized, namely a flat water/air interface in hanging droplet geometry and a solid interface of a glass substrate. The only difference in both realizations is a slightly enhanced self-diffusion coefficient of colloidal particles at the water/air interface at the expense of an increased equilibration time after mounting the sample compared to the solid substrate. The latter is due to a nontrivial regulation of the curvature of the droplet [26]. Video microscopy and digital image analysis provide the position of the particles at a frame rate of about $1 \mathrm{~Hz}$, which is fast compared to the Brownian time scale of $50 \mathrm{~s}$. The colloidal ensemble is described by the Hamiltonian

$$
H=\sum_{i} \frac{p_{i}^{2}}{2 m}+\sum_{i<j} V\left(r_{i j}\right),
$$

with the pair interaction for particles at distance $r$,

$$
\beta V(r)=\frac{3^{3 / 4} \Gamma}{(2 \pi)^{3 / 2}}\left(\frac{a}{r}\right)^{3} .
$$

Here, $\beta=1 / k_{B} T$ with the Boltzmann constant $k_{B}$ and temperature $T$. The distance is given in units of $a$, the average interparticle distance of the triangular lattice. The dimensionless parameter $\Gamma=\beta\left(\mu_{0} / 4 \pi\right)(\chi H)^{2}(\pi \rho)^{3 / 2}$ defines the phase behavior of the system and can be interpreted as an inverse temperature, tuned with the magnetic field $H$. Here, $\mu_{0}$ is the permeability of vacuum, $\chi$ is the magnetic susceptibility of the particles, and $\rho$ is the 2D particle density. The experimental setup is described in detail in Refs. $[16,24,26]$. On the solid substrate, a vacancy is prepared by trapping a colloid with an optical tweezer $(20 \mathrm{~mW}, 100 \times$ tweezer 
objective from above the sample, NA $0.73, \mathrm{Ar}^{+}$-laser) and pulling it alongside a lattice line out of the field of view (at least more than 15 lattice spacings). Correspondingly, an interstitial is created by pulling a particle from the far field into the center of the field of view of an otherwise defect-free crystal. Samples confined at the water/air interface offer additionally the possibility to shoot particles out of the interface by light pressure with a strong laser pulse $(500 \mathrm{~mW})$. Since the sound velocity is of the order of a few $\mathrm{mm} / \mathrm{s}$, the distorted lattice relaxes rapidly and measurements are started after about a minute. Positional data were taken at three different interactions strength, $\Gamma=120,140,156$, with more than 3000 configurations in the case of $\Gamma=120$ and 156 and more than 20000 configurations in the case of $\Gamma=140$ for both interstitials and vacancies. For $\Gamma=140$, the creation of the defect was repeated frequently, annealing the crystal and equilibrating the systems in between for several days up to a few weeks.

\section{DEFECT CLASSIFICATION AND PATTERNS}

In equilibrium, the vacancies and interstitials exist in different, almost degenerate states with different topologies [27,28]. The most frequently appearing vacancy and interstitial states are shown in Fig. 1, and we classify the defects according to their point groups in two dimensions. $C_{n}$ denotes the cyclic group with $n$-fold rotational symmetry, whereas $D_{n}$ is the dihedral group with additionally $n$ mirror axes. The upper left index denotes vacancies or interstitials, and the upper right index counts the number of dislocations involved in the defect. In two dimensions, a dislocation is a pair of fivefold(colored orange in Fig. 1) and sevenfold- (colored green) coordinated particles characterized by a Burgers vector. The neighbor numbers in the vicinity of the defect centers are determined using a Voronoi construction [29]. The dissociation
TABLE I. Classification of interstitial $(I)$ and vacancy $(V)$ states based on the number of particles with four, five, seven, and eight neighbors in the vicinity of the defect.

\begin{tabular}{lccccc}
\hline \hline Defect & $i$ & no. 4 & no. 5 & no. 7 & no. 8 \\
\hline${ }^{I} D_{2}^{0}$ & 1 & 1 & 0 & 2 & 0 \\
${ }^{I} D_{2}^{2}$ & 2 & 0 & 2 & 2 & 0 \\
${ }^{I} D_{3}^{3}$ & 3 & 0 & 3 & 3 & 0 \\
${ }^{I} D_{2}^{4}$ & 4 & 0 & 4 & 4 & 0 \\
${ }^{V} D_{2}^{0}$ & 1 & 0 & 2 & 0 & 1 \\
${ }^{V} C_{2}^{2} /{ }^{V} D_{2}^{2}$ & 2 & 0 & 2 & 2 & 0 \\
${ }^{V} D_{3}^{3}$ & 3 & 0 & 3 & 3 & 0 \\
${ }^{V} D_{2}^{4}$ & 4 & 0 & 4 & 4 & 0 \\
\hline \hline
\end{tabular}

of dislocations is known to drive the melting transition in two dimensions [8]. The most frequent defect configurations are listed in Table I and the index $i$ is introduced to label the defects in the formula below and for the computations of transition rates. Configurations with larger numbers of dislocations exist and are numbered with others $(i=0)$. Note that the number of such defects vanishes for large $\Gamma$ (see the dotted lines in Fig. 10).

Interestingly, the typical symmetry of the configuration consisting of two dislocations (second column in Fig. 1) is not the same for interstitials and vacancies. To quantify the relative probability of dihedral and cyclic configurations, we introduce the following procedure. We connect the fivefold particles with a line and the sevenfold ones too, and we measure the angle $\alpha$ between the two axis. For $\alpha=\pi / 2 \pm \pi / 8$, the pattern is classified as rhombic ( $D_{2}$ symmetry) and otherwise classified as cyclic ( $C_{2}$ symmetry). Figure 2 shows the probability density as a heat map for vacancies (a) and interstitials (b). Red
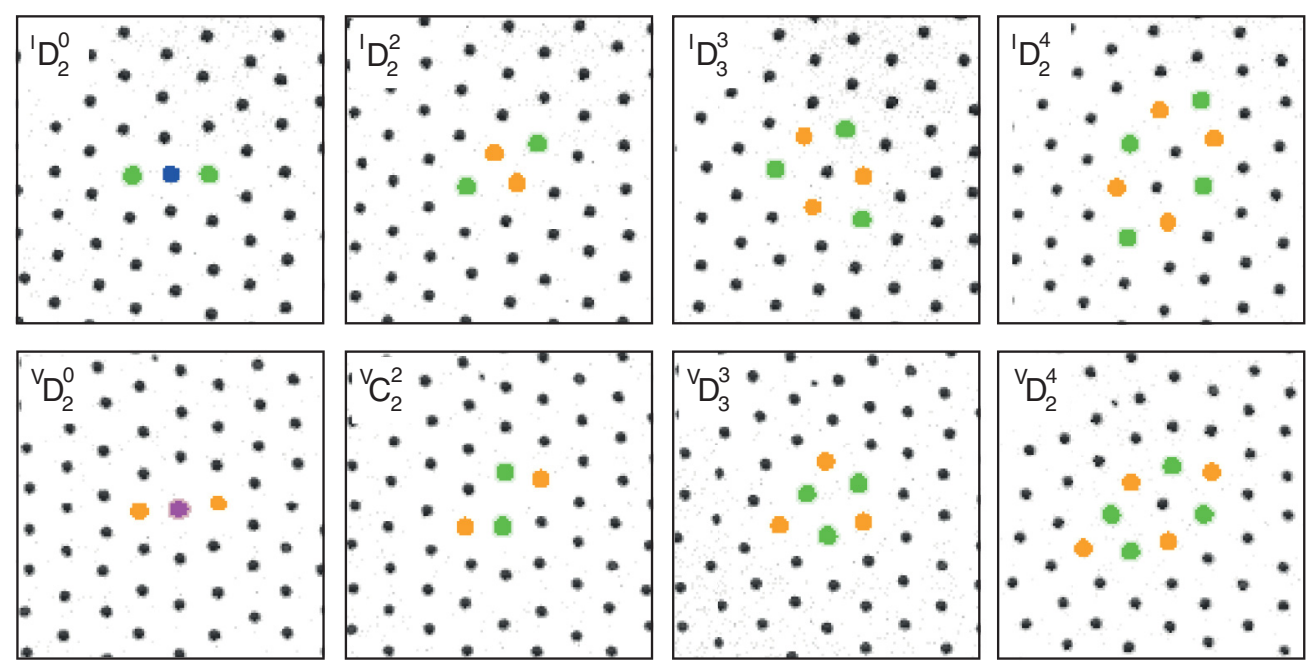

FIG. 1. (Color online) Typical snapshots of the colloidal crystal obtained from the experiment containing an interstitial (upper row) and a vacancy (lower row), respectively. The motion of the defects consists of a sequence of transitions between states with different symmetries given by the dihedral $D_{n}$ and cyclic $C_{n}$ point groups in two dimensions. The upper left index denotes interstitial vs vacancies and the upper right counts the numbers of sevenfold particles (in the case of interstitials) and fivefold particles (in the case of vacancies). Particles with four, five, seven, and eight neighbors are shown in blue (gray), orange (light gray), green (medium gray), and purple (dark), respectively, and particles with six neighbors are shown in black (small dots). Note that the symmetry of the most frequent low-temperature configurations (second column) differ for interstitials and vacancies. The vacancies show only cyclic symmetry for low temperatures. 

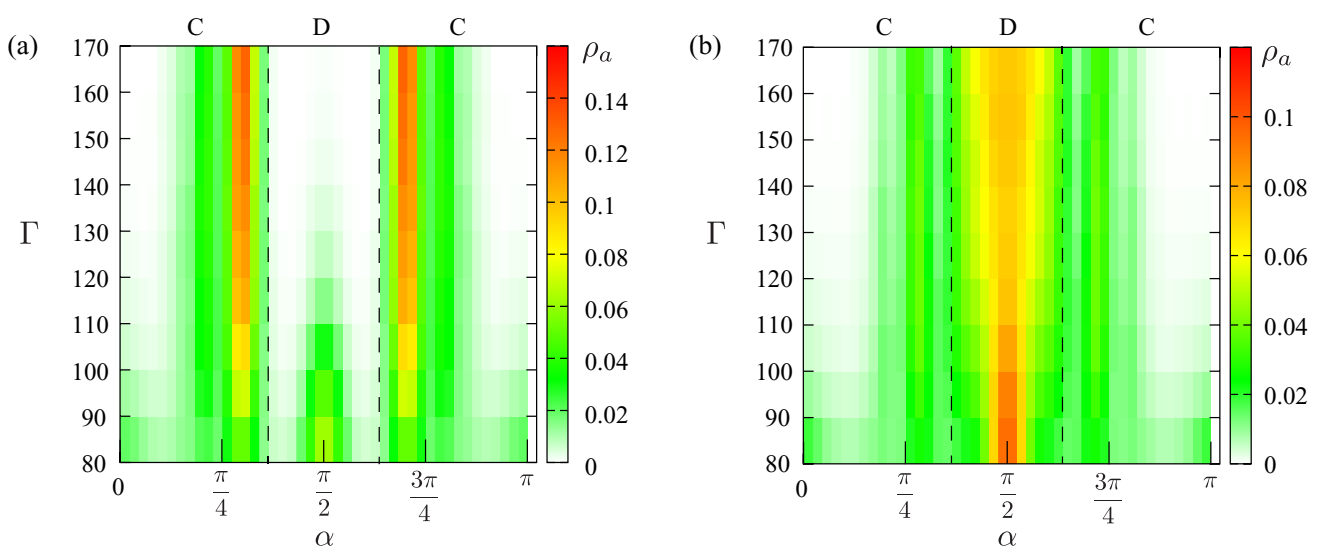

FIG. 2. (Color online) Probability density of the angle $\alpha$ between the 5-5 and 7-7 bonds for vacancies (left) and interstitials (right) consisting of two dislocations for different values of $\Gamma$. Red (dark) indicates high probabilities. For $\alpha=\pi / 2$ the symmetry is dihedral, $D_{2}$. The vacancies are peaked at $\alpha \approx 90^{\circ} \pm 38^{\circ}$ for low temperatures (large $\Gamma$ ) with cyclic symmetry $C_{2}$.

corresponds to large probabilities and green to low ones. For $\Gamma \geqslant 120$, the distribution is sharply peaked at about $\alpha \approx \pi / 2$ for the vacancies, and the probability of the $D_{2}$ symmetry tends to zero. The ratio for $D_{2}$ symmetry increases to $30 \%$ at $\Gamma=80$. Having this in mind, we keep the notation ${ }^{V} C_{2}^{2}$ for vacancies consisting of two dislocations in the following plots for clarity. For interstitials [Fig. 2(b)] the distribution is peaked at $\alpha \approx \pi / 2$ corresponding to dihedral symmetry $D_{2}$ for all interaction strength. Nonetheless, the distribution widens for lower temperatures, which might indicate a separate configuration $\mathrm{C}_{2}$. Using the same cutoff as for vacancies, the ratio varies form $50 \%: 50 \%$ at $\Gamma=80$ to $75 \%{ }^{I} D_{2}$ and $25 \%$ ${ }^{I} C_{2}$ at $\Gamma=170$.

To visualize the configurations with respect to the underlying lattice of the crystal, Figs. 3 and 4 show the probabilities of positions of particles with respect to the defect centers. All patterns reflect the symmetry of the crystal, but the relative positions of particles with four, five, and seven neighbors for the interstitials are arranged in particular patterns shown in Fig. 3, and the five-, six-, and eight-neighbored particles of the vacancies are shown in Fig. 4. Remarkably, the positions of the fivefold-coordinated particles in an interstitial defect and the positions of sevenfold-coordinated particles are all degenerate within the 12 points in a starlike pattern.

\section{EQUILIBRIUM DEFECT POPULATIONS}

Following a trajectory $\mathbf{x}(t)$ of the system in experiment, one can use the classification above to identify a trajectory of states. Here, $\mathbf{x}(t)$ denotes the configuration of the system, including the positions of all particles, at time $t$. In computer simulations, $\mathbf{x}(t)$ is the sequence of Monte Carlo configurations. For illustration, a typical trajectory taken from computer simulations is depicted in Fig. 5. From the trajectory, we determine the probability $P_{i}$ of finding the defect in state $i$ with

$$
P_{i}=\left\langle h_{i}[\mathbf{x}(t)]\right\rangle=\lim _{\tau \rightarrow \infty} \frac{1}{\tau} \int_{0}^{\tau} d t^{\prime} h_{i}\left[\mathbf{x}\left(t^{\prime}\right)\right] .
$$

The indicator function $h_{i}(\mathbf{x})$ is defined as

$$
h_{i}(\mathbf{x})= \begin{cases}1 & \text { if } \mathbf{x} \text { is in state } i, \\ 0 & \text { otherwise. }\end{cases}
$$

The probabilities are normalized to $\sum_{i} P_{i}=1$ for vacancies and interstitials separately. The population $P_{i}$ equals the fraction of time the defect spends in state $i$ in equilibrium. This probability is estimated from a finite number of configurations,

$$
P_{m}=\bar{h}=\frac{1}{N} \sum_{i=1}^{N} h_{m}[x(i \Delta t)] .
$$

The configurations are sampled in experiment and simulation at constant time intervals. These configurations may therefore be correlated, and the error from this estimation, including correlated events, is

$$
\sigma^{2}=\left\langle(\bar{h}-\langle h\rangle)^{2}\right\rangle=\frac{1}{N^{2}} \sum_{i j}\langle\delta h(i) \delta h(j)\rangle,
$$

where $\langle h\rangle$ is the ensemble average of $h$, and $\delta h(i)=h[x(i \Delta t)]-\langle h\rangle$. The correlation function $\langle\delta h(i) \delta h(j)\rangle$ can be rewritten as

$$
\begin{aligned}
H(|i-j|) & =\langle\delta h(i) \delta h(j)\rangle=\langle h(i) h(j)\rangle-\langle h\rangle^{2} \\
& =C_{m, m}\left(\Delta t_{i-j}\right)-\langle h\rangle^{2} .
\end{aligned}
$$

Here, $C_{m, m}$ are the autocorrelation functions as depicted in Figs. 6-9. Note that in equilibrium, $H(i-j)=H(|i-j|)$ and $H(0)=\langle h\rangle(1-\langle h\rangle)$. Inserting Eq. (7) into Eq. (6) with $t=|i-j|$, we find

$$
\sigma^{2}=\frac{1}{N}\left(2 \sum_{t=0}^{N-1} H(t)-H(0)\right)-\frac{2}{N^{2}} \sum_{t=1}^{N-1} t H(t) .
$$

In the limit of large $N$, the last term, which scales quadratically, and the constant term $H(0)$ can be neglected, and the error can be estimated from

$$
\sigma^{2} \approx \frac{2}{N} H(0) \sum_{t=0}^{N-1} \frac{H(t)}{H(0)}=\frac{\langle h\rangle(1-\langle h\rangle)}{N} \sum_{t=0}^{N-1} \frac{H(t)}{H(0)} .
$$

Note that the term $\frac{\langle h\rangle(1-\langle h\rangle)}{N}=\sigma_{0}^{2}$ is the error from $N$ uncorrelated measurements. In a general trajectory, correlations increase the error by $2 \sum_{t=0}^{N-1} \frac{H(t)}{H(0)}$. This factor can be 
(a)
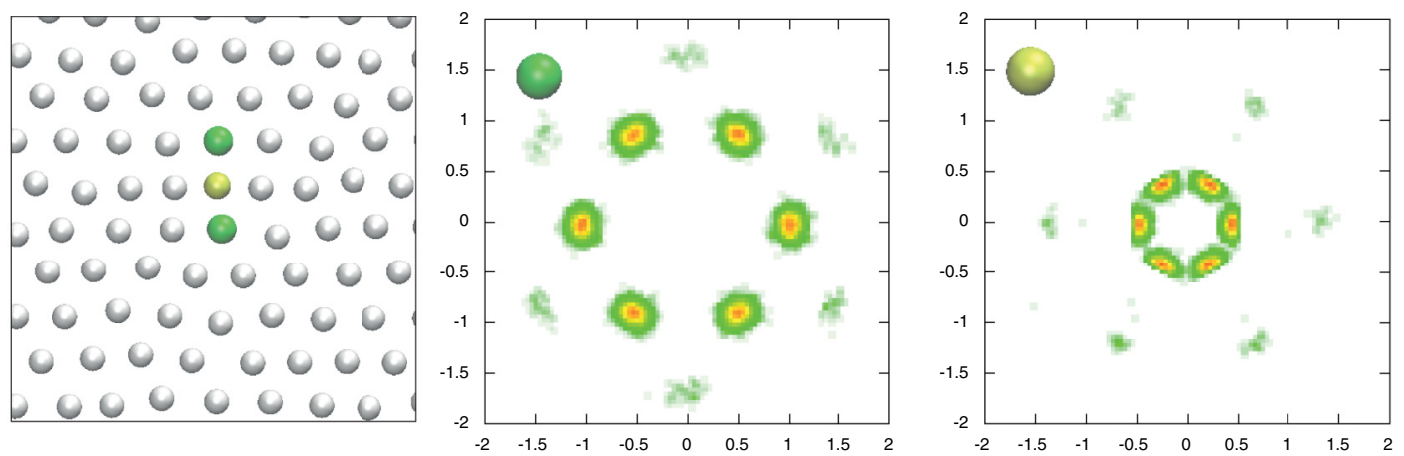

(b)
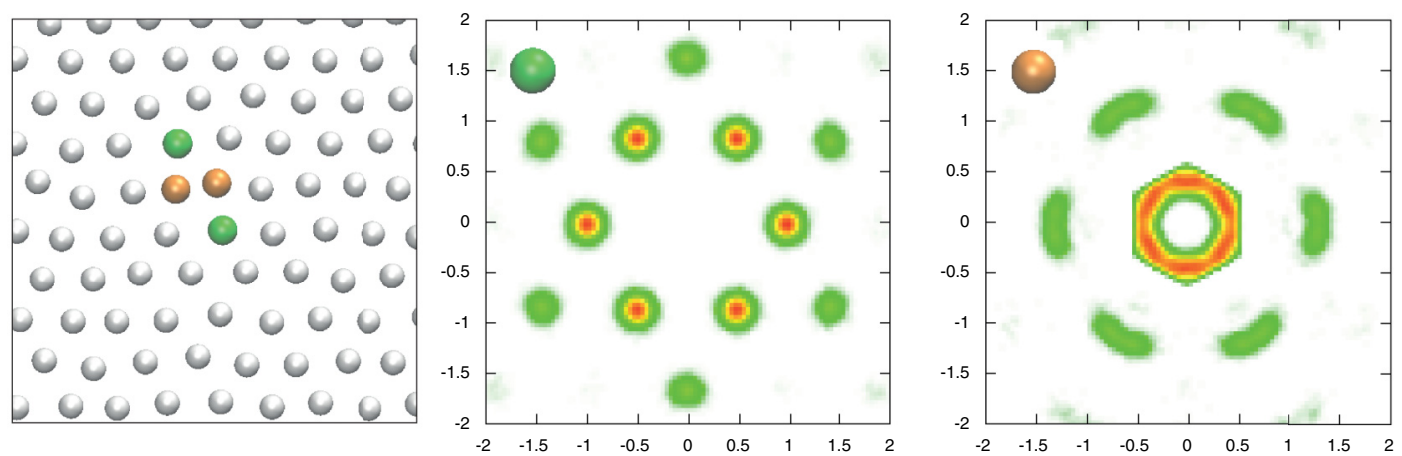

(c)
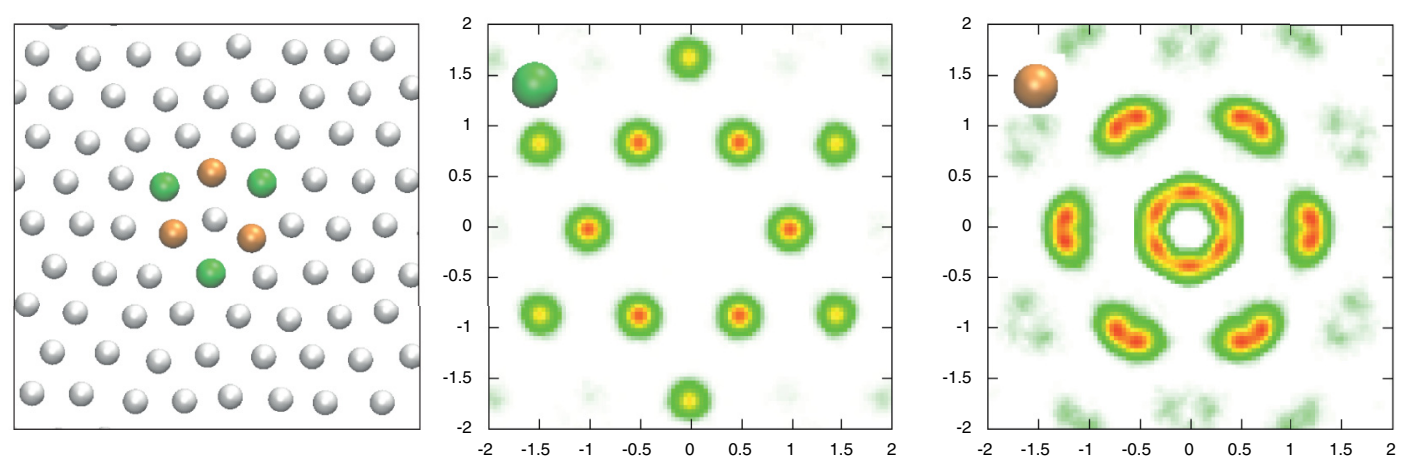

FIG. 3. (Color online) Relative positions of fourfold-, fivefold-, and sevenfold-coordinated particles in the vicinity of an interstitial defect with respect to the defect position on the underlying triangular lattice from computer simulations. The configurations (left column) ${ }^{I} D_{2}^{0}$ (a), ${ }^{I} D_{2}^{2}$ (b), and ${ }^{I} D_{3}^{3}$ (c) consist of at least two fivefold-coordinated particles [green (medium gray)]. The ${ }^{I} D_{2}^{0}$ state consists of an additional fourfold-coordinated particle [yellow (light)] while the ${ }^{I} D_{2}^{2}$ and ${ }^{I} D_{3}^{3}$ states consist of additional sevenfold-coordinated particles [orange (light gray)]. The relative positions of fivefold-coordinated (middle column) and fourfold- and sevenfold-coordinated particles (right column) are measured with respect to the closest lattice point of the defect center and depicted as a heat map. Red indicates large probability and white low probabilities. Clearly, the fivefold-coordinated particles always occupy the same 12 lattice points with different weights depending on the state. The fivefold- and sevenfold-coordinated particles also follow different patterns.

associated with the correlation time $t_{c}$ in the system by

$$
\sum_{t=0}^{N-1} \frac{H(t)}{H(0)} \approx \frac{1}{\Delta t} \frac{1}{H(0)} \int_{0}^{\infty} H(t) d t=\frac{t_{c}}{\Delta t}
$$

Here, the correlation time is $t_{c}=\frac{1}{H(0)} \int_{0}^{\infty} H(t) d t$ and $\Delta t$ is the sampling interval. Combining all this, the error from correlated trajectories can be written as the uncorrelated error multiplied by the correlation time,

$$
\sigma^{2}=\sigma_{0}^{2} \frac{2 t_{c}}{\Delta t} .
$$

In the experiments, the trajectory is sampled every $\Delta t=0.92 \mathrm{~s}$. The number of measurements differs considerably for the various $\Gamma$ for vacancy and interstitials. In particular, for inter- stitials $N_{\Gamma=120}^{I}=670$ and $N_{\Gamma=140}^{I}=31500$ and for vacancies $N_{\Gamma=120}^{V}=4950, N_{\Gamma=140}^{V}=23700$, and $N_{\Gamma=156}^{V}=9900$. The correlation time for different species ranges from $t_{c}=20$ to 40 .

In the computer simulations, the trajectory is a sequence taken from Monte Carlo updates. The number of particles is $N_{p}=26 \times 30+1=781$ for interstitials and $N_{p}=779$ for vacancies. Interactions are cut off at a radius $r_{c}=8 a_{0}$, where $a_{0}$ is the lattice spacing. The displacement in each Monte Carlo step is chosen such that the average acceptance rate is approximately 0.5. Sequences are sampled every 800000 Monte Carlo steps, which corresponds to approximately $1 \mathrm{~s}$ in real time. For all parameters, the total number of measurements is $N=750000$.

Populations of interstitial and vacancy states obtained from computer simulations for $\Gamma$ ranging from $\Gamma=100$ to 170 
(a)
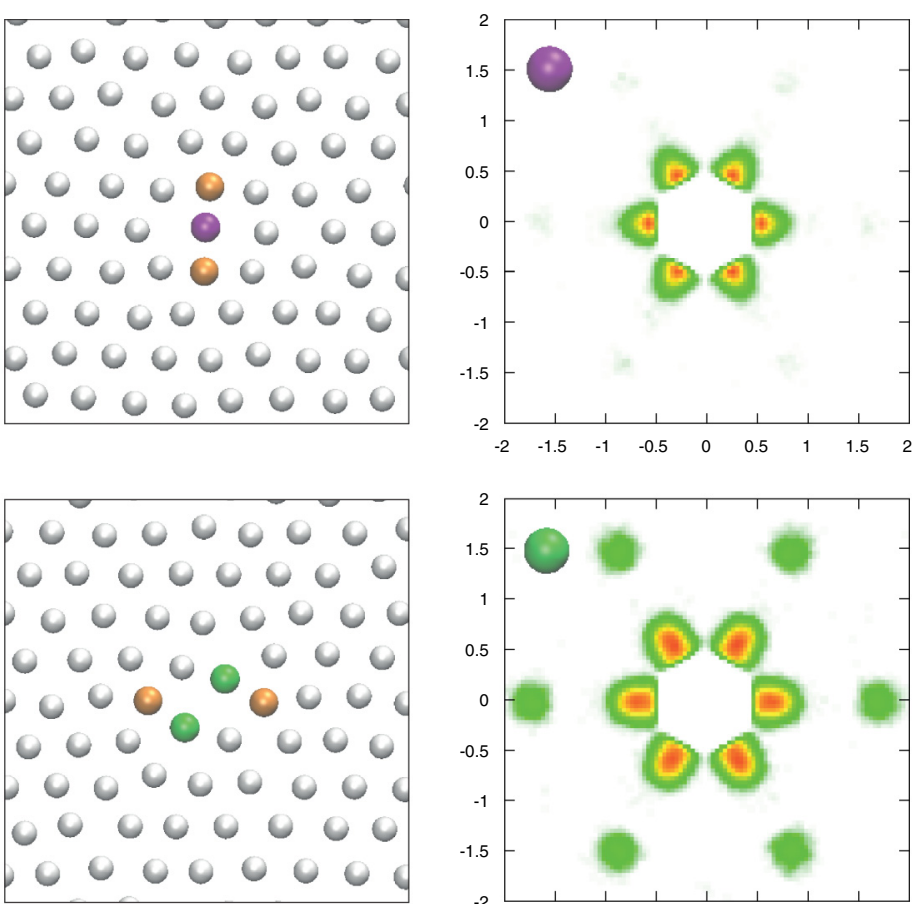

(c)
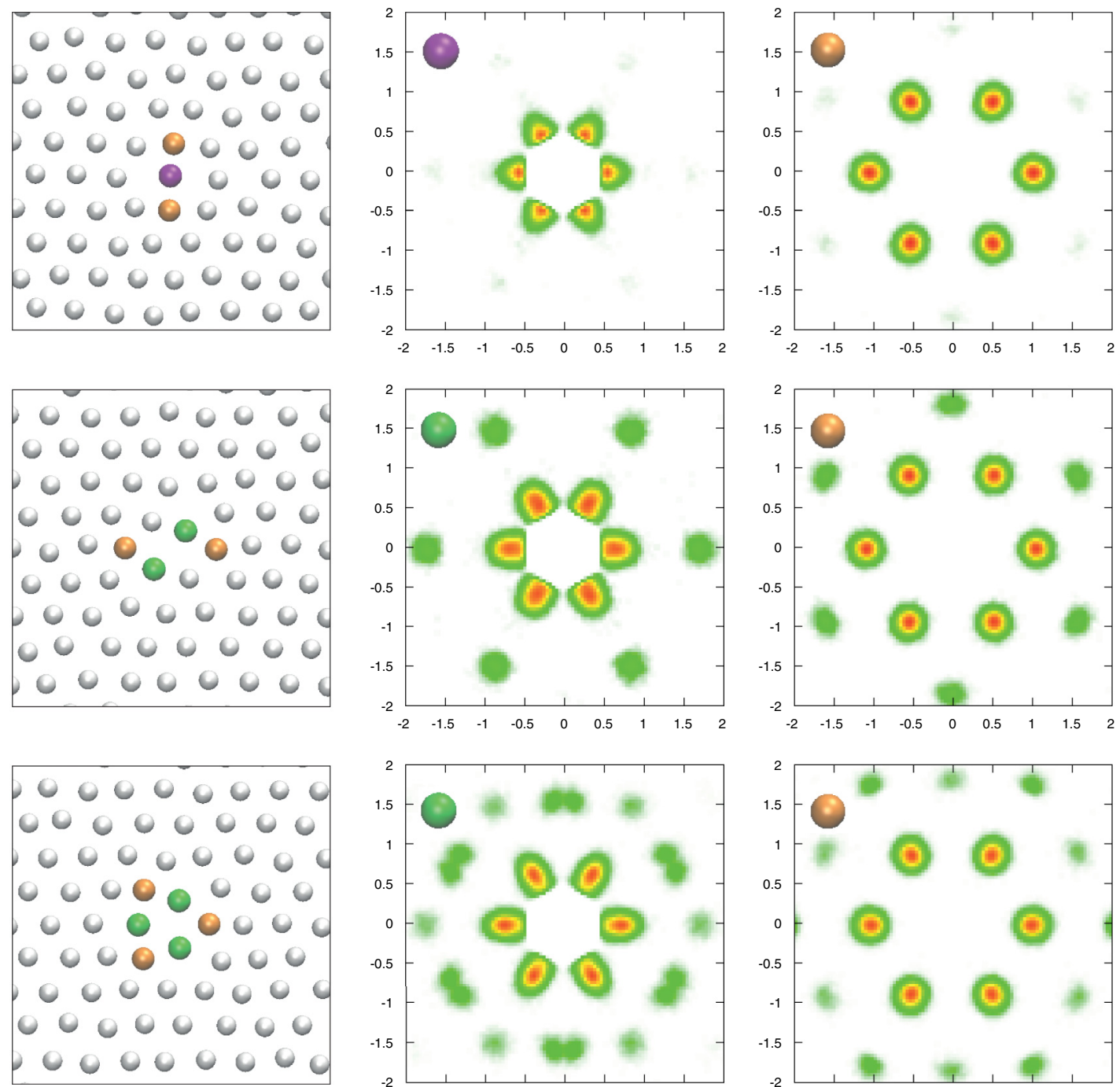

(b)

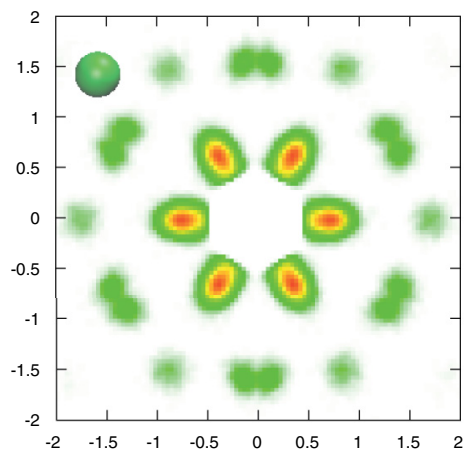

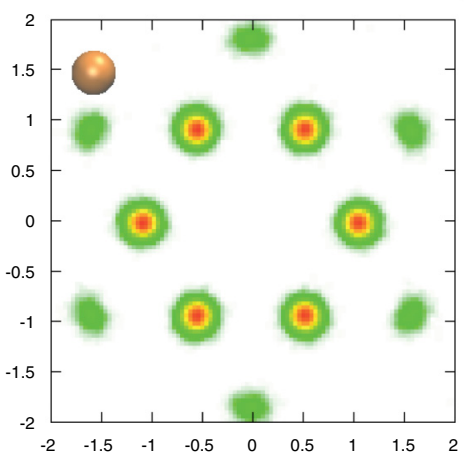

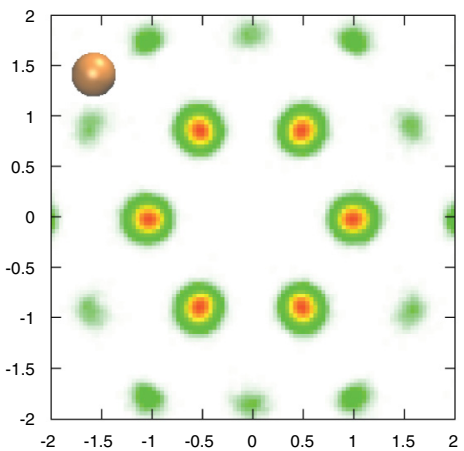

FIG. 4. (Color online) Relative positions of fivefold-, sevenfold-, and eightfold-coordinated particles in the vicinity of a vacancy defect with respect to the defect position on the underlying triangular lattice from computer simulations as described in the caption of Fig. 3. A vacancy in state ${ }^{V} D_{2}^{0}$ (a), ${ }^{V} C_{2}^{2}$ (b), or ${ }^{V} D_{3}^{3}$ (c) consists of at least two sevenfold-coordinated particles (orange) and either a single eightfold-coordinated (violet) particle (a) or additional fivefold-coordinated particles (green) (b) and (c). In all states, the sevenfold-coordinated particles are found at the same 12 positions (right) while the eightfold- and fivefold-coordinated particles are found at various other positions.

are shown in Fig. 10. The defect populations determined experimentally for interstitials and vacancies at $\Gamma=120,140$, and 156 are in good agreement. For the vacancies, the small overpopulation for $\Gamma<140$ of ${ }^{V} C_{2}^{2}$-type with the lowest symmetry (red squares) is attributed to a tiny shear within the sample since vacancies were mainly created at the water/air interface. This interface is less stable compared to the solid substrate, but vacancies can be created with fewer perturbations by pushing particles out of the interface using the light pressure of the laser pulse.

\section{FREE ENERGY OF DEFECT STATES}

The probability of finding the defect in state $i$ is related to the free energy of the state, which consists of an energy and an entropy contribution. For convenience, we introduce the reduced energy $W(\mathbf{x})=\frac{3^{3 / 4}}{2 \pi^{3 / 2}} \sum_{i<j}\left(a / r_{i j}\right)^{3}$, which is related to the potential energy $V(\mathbf{x})$ by $\beta V(\mathbf{x})=\Gamma W(\mathbf{x})$. The probability density in configuration space for a given temperature and volume is given by

$$
\rho(\mathbf{x})=Z^{-1} e^{-\Gamma W(\mathbf{x})}
$$

with the partition function $Z=\int e^{-\Gamma W(\mathbf{x})} d \mathbf{x}$. The probability of finding the defect in state $i$ can then be written as an ensemble average,

$$
P_{i}=\frac{\int e^{-\Gamma W(\mathbf{x})} h_{i}(\mathbf{x}) d \mathbf{x}}{\int e^{-\Gamma W(\mathbf{x})} d \mathbf{x}}=\frac{Z_{i}}{Z}=e^{-\Gamma \Delta F_{i}},
$$

where $Z_{i}=\int e^{-\Gamma W(\mathbf{x})} h_{i}(\mathbf{x}) d \mathbf{x}$ is the partition function restricted to state $i$. Thus, $\Delta F_{i}=F_{i}-F$ is the difference between the free energy $F_{i}=-\Gamma^{-1} \ln Z_{i}$ of configuration $i$ and the free energy $F=-\Gamma^{-1} \ln Z$ of the system. According to basic statistical mechanics, the free energy $F_{i}$ is the sum of 


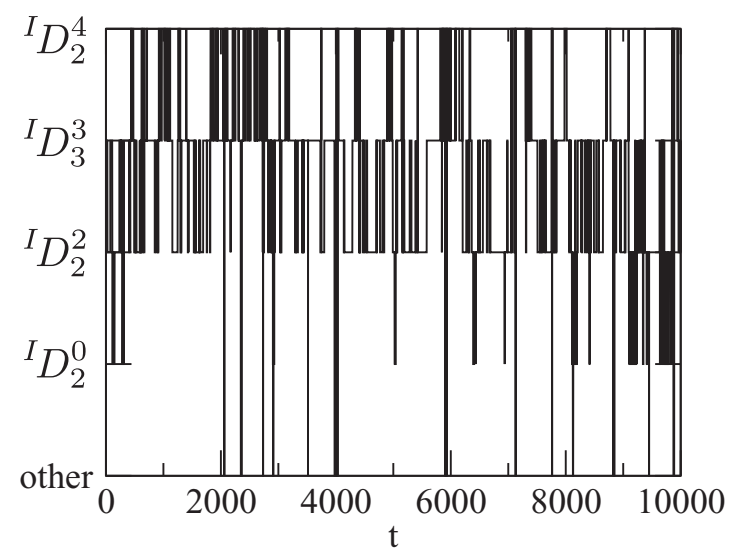

FIG. 5. Typical trajectory of the state as a function of time (in units of Monte Carlo time steps) of an interstitial at $\Gamma=160$. The state is identified in each time step from the neighbor numbers of particles in the vicinity of the defect according to Table I.

an energetic and an entropic contribution,

$$
F_{i}=\langle W\rangle_{i}-\frac{S_{i}}{\Gamma}
$$

Here, $\langle W\rangle_{i}$ is the average energy given that the system is in state $i$, and $S_{i}$ is the entropy of state $i$.

For sufficiently large values of $\Gamma$, at which configurations belonging to state $i$ can be viewed as small fluctuations about a local energy minimum, the energetic and entropic contributions to the free energy can be computed analytically. In this regime, the energy of each state $i$ is approximated as quadratic
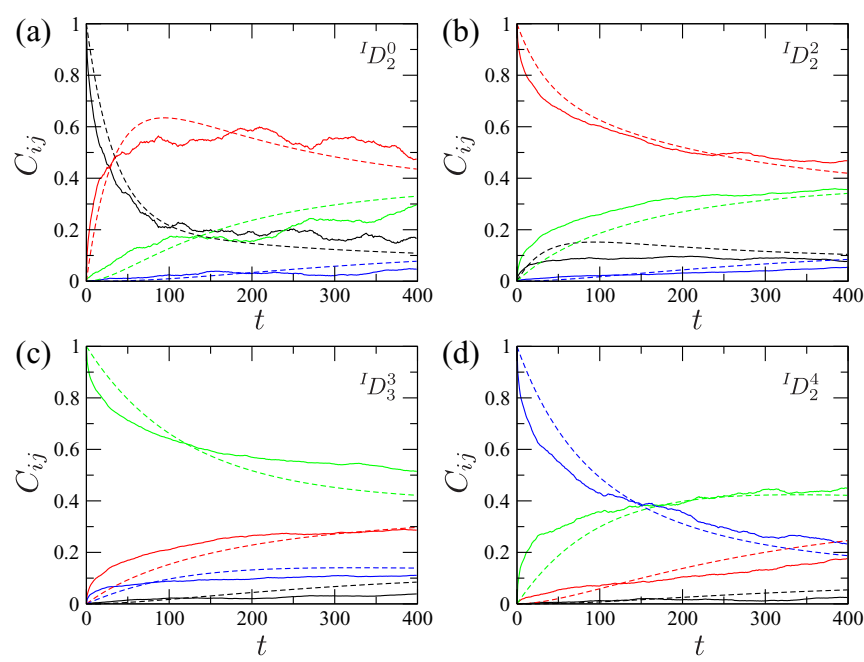

FIG. 6. (Color online) Time correlation functions $C_{i j}(t)$ for interstitials obtained from experiment (solid lines) in comparison to the result of the optimization procedure of $K_{i j}$ (dashed) for $\Gamma=140$. The symbols in the top right corner of each panel indicate the initial state $j$ and colors indicate the final state $i$, where black corresponds to ${ }^{I} D_{2}^{0}$, red to ${ }^{I} D_{2}^{2}$, green to ${ }^{I} D_{3}^{3}$, and blue to ${ }^{V} I_{2}^{4}$. For instance, the black lines in the top left panel represent the probability to find the defect in the ${ }^{I} D_{2}^{0}$ configuration at time $t$ given that it was in ${ }^{I} D_{2}^{0}$ at time $t=0$, while the red lines represent the probability to find it in ${ }^{I} D_{2}^{2}$ at time $t$.
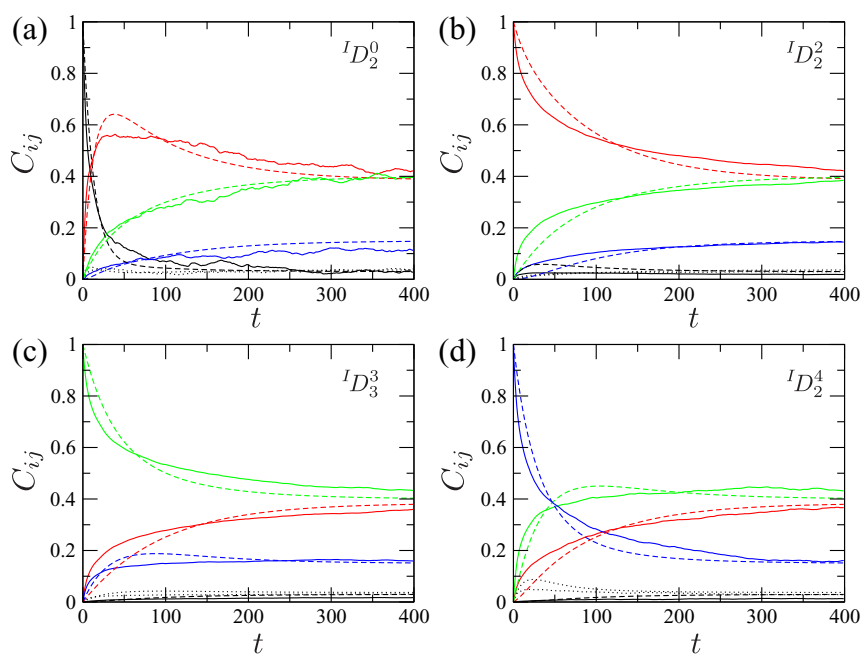

FIG. 7. (Color online) Correlation functions $C_{i j}(t)$ for interstitials obtained from simulation (solid lines) and from the optimization procedure of $K_{i j}$ (dashed). Parameters and colors as in Fig. 6 .

function centered at the minimum energy configuration $\mathbf{x}_{i}^{0}$,

$$
W_{i}(\mathbf{x})=W\left(\mathbf{x}_{i}^{0}\right)+\frac{1}{2} \mathbf{u}_{i} \mathcal{D}_{i} \mathbf{u}_{i},
$$

where $\mathbf{u}_{i}=\mathbf{x}-\mathbf{x}^{0}$ is the displacement from the minimum and $\mathcal{D}_{i}$ is the matrix of second derivatives of $W$ evaluated at $\mathbf{x}_{i}^{0}$. The partition function of state $i$ is then given by $Z_{i}=e^{-\Gamma W\left(\mathbf{x}_{i}^{0}\right)} \sqrt{\frac{(2 \pi)^{n}}{\Gamma^{n} \operatorname{det} D_{i}}}$, where $n$ is the number of degrees of freedom. From this expression, it follows that the free-energy difference $\Delta F_{k l}=F_{k}-F_{l}$ between two states $k$ and $l$, which determines the relative population $P_{k} / P_{l}$, can be expressed as

$$
\begin{aligned}
\Delta F_{k l} & =-\frac{1}{\Gamma} \ln \frac{P_{k}}{P_{l}} \\
& =W\left(\mathbf{x}_{k}^{0}\right)-W\left(\mathbf{x}_{l}^{0}\right)-\frac{1}{\Gamma} \frac{1}{2} \ln \frac{\operatorname{det} \mathcal{D}_{k}}{\operatorname{det} \mathcal{D}_{l}} \\
& =\Delta W_{k l}-\frac{1}{\Gamma} \Delta S_{k l} .
\end{aligned}
$$
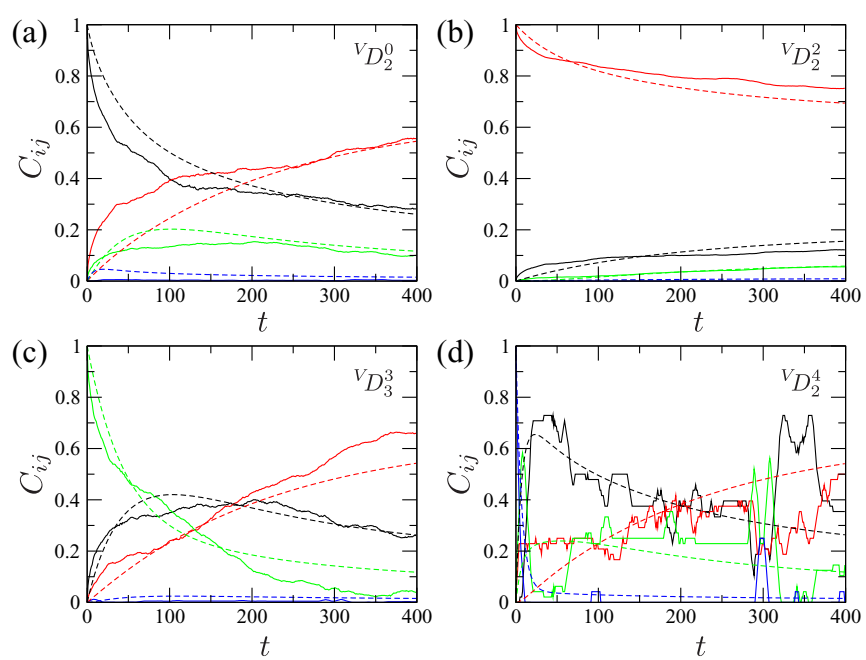

FIG. 8. (Color online) Correlation functions $C_{i j}(t)$ for vacancies obtained from experiment (solid line) and from the optimization procedure of $K_{i j}$ (dashed). Parameters and colors as in Fig. 6. 

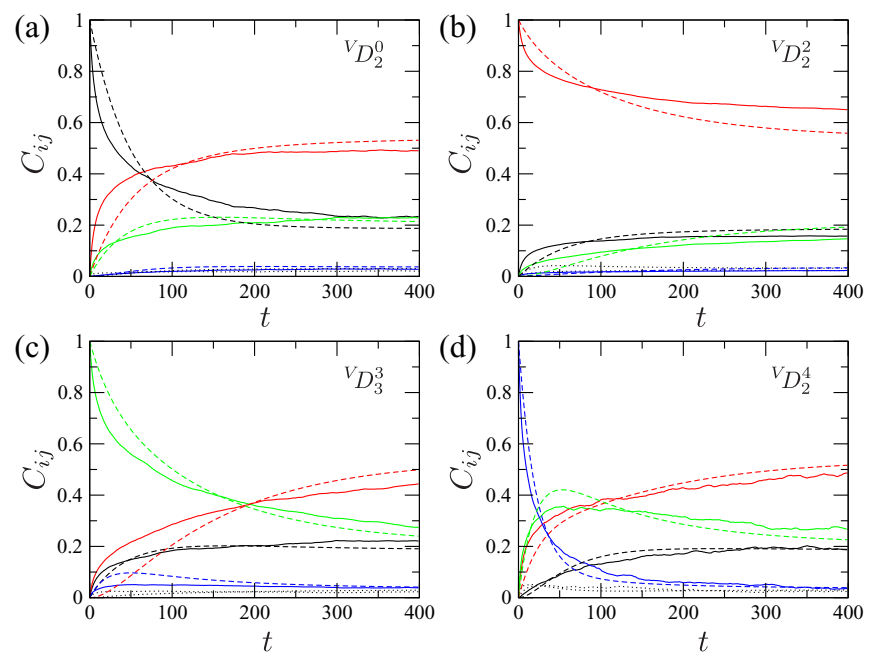

FIG. 9. (Color online) Correlation functions $C_{i j}(t)$ for vacancies obtained from simulation (solid line) and from the optimization procedure of $K_{i j}$ (dashed). Parameters and colors as in Fig. 6.
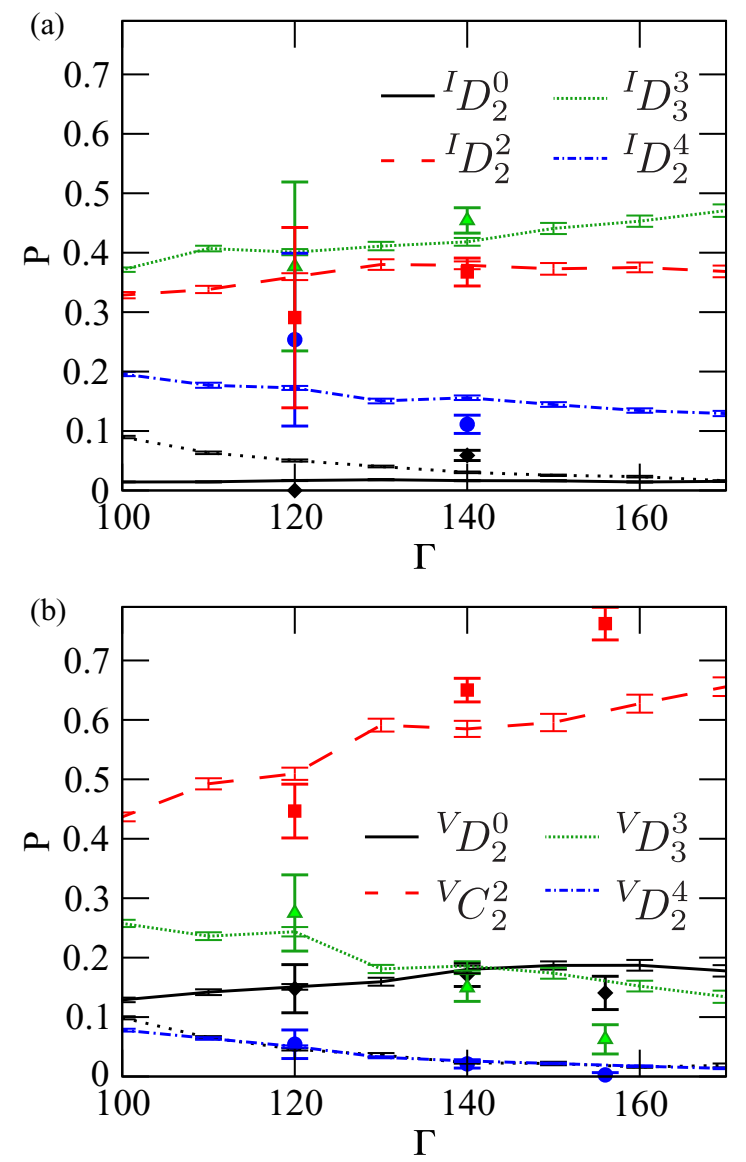

FIG. 10. (Color online) Equilibrium populations of (a) interstitial and (b) vacancy states identified based on Table I as a function of $\Gamma$ from experiments (filled symbols) and simulations (solid lines). The dotted line denotes the probability to find the defect in a state not given in the table that is negligible for higher interaction strength $\Gamma$. Error bars depict the statistical error from finite numbers of measurements taking into account the correlations between the individual configurations.
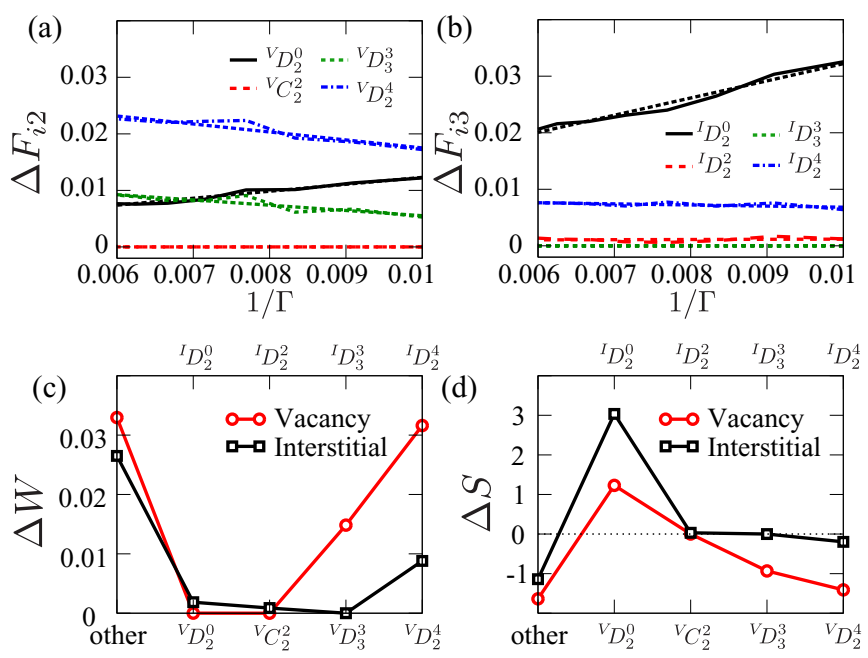

FIG. 11. (Color online) Free-energy difference $\Delta F_{k l}$ as a function of $1 / \Gamma$ for vacancies (a) and interstitials (b). The dashed lines are linear fits to the data obtained from computer simulations. According to Eq. (16), the intercepts of these functions corresponds to energy differences $\Delta W_{k l}$ shown in panel (c) for interstitials (black squares) and vacancies (red circles). The energies are given with respect to the lowest energy state, which is ${ }^{V} C_{2}^{2}$ and ${ }^{I} D_{3}^{3}$ for vacancies and interstitials, respectively. The slopes of the lines in panels (a) and (b) yield the entropy differences $\Delta S_{k l}$ shown in panel (d) for interstitials (black) and vacancies (red).

Thus, the free-energy difference $\Delta F_{k l}=F_{k}-F_{l}$ depends linearly on $1 / \Gamma$. The intercept of this function with the $y$ axis then yields the energy difference $\Delta W_{k l}=W\left(\mathbf{x}_{k}^{0}\right)-W_{l}\left(\mathbf{x}_{l}^{0}\right)$ between states $k$ and $l$, and the slope equals the entropy difference $\Delta S_{k l}$. As shown in Fig. 11, this linear behavior is indeed observed in our simulations. Figure 11 also shows the energy and entropy differences obtained by linear fits of Eq. (16) to the simulation results. As can be inferred from the figure, the symmetric defect configurations ${ }^{V} D_{2}^{0}$ and ${ }^{I} D_{2}^{0}$ have a positive entropy with respect to the respective lowest-energy states, leading to a negative slope of the free energy versus $1 / \Gamma$ curves shown as black lines in Figs. 11(a) and 11(b). Note that while the energy difference between states is small for all $\Gamma$, the entropy versus energy ratio may change dramatically as a function of $\Gamma$. This positive entropy difference for the symmetric defect configurations causes an inversion of the population order for lower values of $\Gamma$.

\section{DEFECT KINETICS}

On a coarse level, neglecting microscopic details, the motion of a defect can be viewed as a sequence of transitions between discrete states. This type of dynamics can be mapped onto a Markov state model governed by the master equation

$$
\frac{d P_{i}(t)}{d t}=\sum_{j \neq i}\left[K_{i j} P_{j}(t)-K_{j i} P_{i}(t)\right] .
$$

Here, $P_{i}(t)$ is the probability of finding the defect in state $i$ at time $t$, and $K_{i j}$ is the rate constant for transitions from state $j$ to state $i$. The general solution of the master equation can be 
TABLE II. Rate constant matrix $K_{i j}$ for $\Gamma=140$ obtained from experiment and simulation using the optimization of Eq. (25).

\begin{tabular}{|c|c|c|c|c|c|c|c|c|c|}
\hline \multicolumn{5}{|c|}{ Experiment - Interstitials $\left(\times 10^{2}\right)$} & \multicolumn{5}{|c|}{ Experiment - Vacancies $\left(\times 10^{2}\right)$} \\
\hline-1.5014 & 0.0000 & 0.2827 & 0.4473 & 0.7714 & -1.4511 & 0.1469 & 1.0814 & 0.2227 & 0.0000 \\
\hline 0.0040 & -2.1958 & 2.1918 & 0.0000 & 0.0000 & 0.0694 & -0.9595 & 0.5917 & 0.2984 & 0.0000 \\
\hline 0.0218 & 0.5257 & -0.8295 & 0.2820 & 0.0000 & 0.1820 & 0.1521 & -0.3341 & 0.0000 & 0.0000 \\
\hline 0.1171 & 0.0000 & 0.2219 & -0.4665 & 0.1275 & 0.0509 & 0.2643 & 0.0000 & -0.5841 & 0.2689 \\
\hline 0.3825 & 0.0000 & 0.0000 & 0.4346 & -0.8172 & 0.0000 & 0.0000 & 0.0000 & 1.0602 & -1.0603 \\
\hline \multicolumn{5}{|c|}{ Simulation - Interstitials $\left(\times 10^{2}\right)$} & \multicolumn{5}{|c|}{ Simulation - Vacancies $\left(\times 10^{2}\right)$} \\
\hline-2.0873 & 0.0000 & 0.5688 & 0.5834 & 0.9351 & -1.7116 & 0.2209 & 0.9771 & 0.2794 & 0.2342 \\
\hline 0.1637 & -1.9832 & 1.6178 & 0.2016 & 0.0000 & 0.0000 & -0.4375 & 0.2625 & 0.1734 & 0.0015 \\
\hline 0.0231 & 0.1631 & -0.3263 & 0.1401 & 0.0000 & 0.0874 & 0.0812 & -0.1686 & 0.0000 & 0.0000 \\
\hline 0.0578 & 0.0000 & 0.1093 & -0.3682 & 0.2012 & 0.0000 & 0.1483 & 0.0000 & -0.3230 & 0.1747 \\
\hline 0.2939 & 0.0000 & 0.0543 & 0.4219 & -0.7701 & 0.1621 & 0.0000 & 0.3248 & 0.7269 & -1.2137 \\
\hline
\end{tabular}

written in matrix and vector notation as

$$
\mathbf{P}(t)=\exp [\mathbf{K} t] \mathbf{P}(0) \text {. }
$$

Here, $\mathbf{P}(t)$ is the vector of probabilities $P_{i}$ at time $t$, and $\mathbf{K}$ is the matrix of transition rate constants $K_{i j}$. While for short times $\mathbf{P}(t)$ depends on the initial conditions $\mathbf{P}(0)$, for long times $\mathbf{P}(t)$ converges to the vector $\mathbf{P}^{\mathrm{eq}}$ of equilibrium populations independent of time and initial conditions,

$$
\mathbf{P}^{\mathrm{eq}}=\lim _{t \rightarrow \infty} \exp [\mathbf{K} t] \mathbf{P}(0) .
$$

For the matrix of rate constants, the condition of detailed balance holds with respect to the equilibrium distribution $\mathbf{P}^{\mathrm{eq}}$,

$$
\frac{P_{i}^{\mathrm{eq}}}{P_{j}^{\mathrm{eq}}}=\frac{K_{j i}}{K_{i j}} .
$$

In addition, the conservation of total probability requires

$$
K_{i i}=-\sum_{j \neq i} K_{j i}
$$

The transition rate constants $K_{i j}$ can be calculated from a trajectory of states with the following procedure. To characterize the time evolution of the system, we introduce the correlation functions

$$
C_{i j}(t)=P(i, t \mid j, 0)=\frac{\left.\left\langle h_{i}[\mathbf{x}(t)] h_{j} \mathbf{x}(0)\right]\right\rangle}{\left\langle h_{j}[\mathbf{x}(0)]\right\rangle} .
$$

The correlation function $C_{i j}(t)$ is the conditional probability of finding the defect in state $i$ at time $t$, given that it was in state $j$ at time $t^{\prime}=0$. The equilibrium probability $P_{i}^{\mathrm{eq}}$ of state $i$ is the large time limit of $P(i, t \mid j, 0)$,

$$
P_{i}^{\mathrm{eq}}=\lim _{t \rightarrow \infty} P(i, t \mid j, 0) .
$$

To obtain this equation, we have used the fact that for long times the state of the system at time $t$ is statistically independent of the state at time $t=0$, i.e., $\left\langle h_{i}(t) h_{j}(0)\right\rangle=$ $\left\langle h_{i}(t)\right\rangle\left\langle h_{j}(0)\right\rangle$. The correlation functions $C_{i j}(t)$ can be easily determined from trajectories obtained in experiments or simulations. This correlation function is then compared to the result of the master equation given the matrix of rate constants $K_{i j}$ with

$$
\hat{C}_{i j}(t)=\left(\exp [\mathbf{K} t] \mathbf{P}_{j}^{\text {init }}\right)_{i} .
$$

Here, the initial vector $\mathbf{P}_{j}^{\text {init }}$ has a 1 in component $j$ while all other components have a value of 0 . This particular choice of initial condition implies that the system is initially in state $j$ with probability 1 as required by the definition of the conditions probability $C_{i j}$.

To determine the matrix of rate constants $K_{i j}$ governing the dynamics of defects, we carry out an optimization procedure that minimizes the difference between the time correlation functions measured in experiments or simulations and those predicted from the solution of the master equation (see Table II). The target function of this optimization is defined as

$$
E(\mathbf{K})=\sum_{i, j} \sum_{l}\left[\hat{C}_{i j}(l \Delta t)-C_{i j}(l \Delta t, \mathbf{K})\right]^{2} .
$$

Here, $C_{i j}(\Delta t)$ denotes the matrix of correlation functions determined from experiment or simulation. The argument $\mathbf{K}$ in the target function $E(\mathbf{K})$ and in the matrix of predicted correlation functions $\hat{C}_{i j}(\Delta t, \mathbf{K})$ emphasizes their dependence on the matrix of rate constants. The matrix of rate constants is optimized to best reproduce the observed time correlation functions. In this optimization procedure, the target function of Eq. (25) is then minimized iteratively. In each step of the iteration, a matrix element $K_{i j}$ is chosen at random and changed by adding a random amount $\Delta k$. To satisfy the constraint Eq. (21), $\Delta k$ is also subtracted from matrix element $K_{i i}$. The step is accepted if the target function has decreased. The iteration is stopped when the target function has not decreased for a certain number of steps. The optimization procedure is initialized with the time derivatives of the time correlation functions $C_{i j}(t)$ evaluated at $t=0$, with $K_{i j}^{\mathrm{TST}}=d C_{i j}(t) /\left.d t\right|_{t=0}$. These initial transition rate constants correspond to the transition state theory estimates obtained for the dividing surfaces defined implicitly by the state classification introduced earlier. In the transition state theory approximation, correlated crossings of the dividing surface are neglected. Note that the optimization procedure described above takes these transient short-time correlations correctly into account.

Transition rate constants obtained with this optimization procedure for vacancies and interstitials are summarized in Fig. 12. The results from computer simulations and experiments are in very good agreement. The results show that the transition rates are not homogeneous. The most dominant transitions are ${ }^{I} D_{2}^{0} \rightarrow{ }^{I} D_{2}^{2}$ for interstitials and ${ }^{V} D_{2}^{4} \rightarrow{ }^{V} D_{3}^{3}$ for 


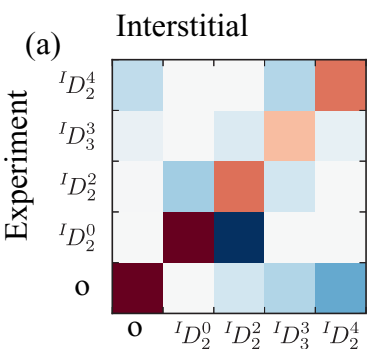

(c)
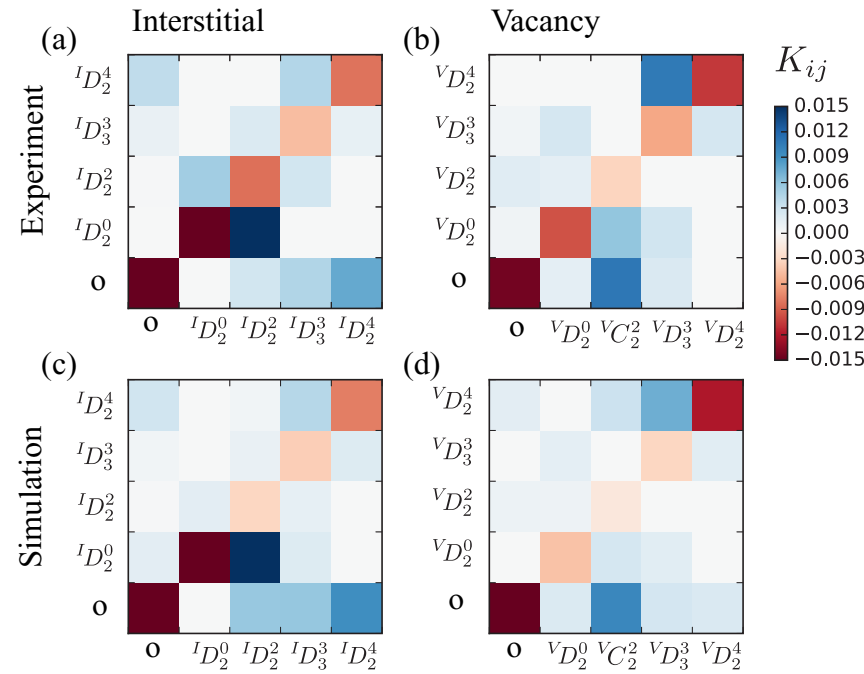

(d)

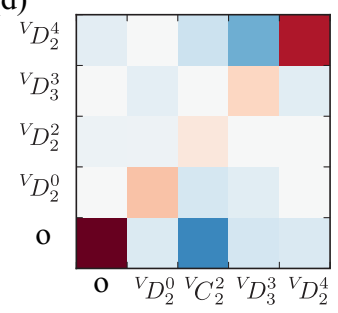

FIG. 12. (Color online) Color-coded optimized rate matrix $\mathbf{K}_{i j}$ for an interstitial (a) and (c) and a vacancy (b) and (d) from a trajectory from experiment (top) and simulation (bottom) at $\Gamma=140$.

vacancies. We also identify several transitions with a vanishing rate (e.g., ${ }^{I} D_{2}^{4} \rightarrow{ }^{I} D_{2}^{0}$ or ${ }^{V} D_{2}^{4} \rightarrow{ }^{V} C_{2}^{2}$ ). We plan to investigate the exact microscopic mechanism for these rare transitions in the future.

In summary, we have shown that geometrical defects such as interstitials and vacancies appear as different topological configurations mainly constructed of two, three, or four dislocations where the Burgers vector cancels. We characterize the configurations by the symmetry of $2 \mathrm{D}$ point groups and show that the symmetries for interstitials and vacancies are not equivalent. The relative equilibrium probabilities of defects vary as a function of the temperature. In the low-temperature limit, the probabilities of ${ }^{I} D_{2}^{2}$ and ${ }^{I} D_{3}^{3}$ symmetries are largest for interstitials, while vacancies are predominantly in the ${ }^{V} C_{2}^{2}$ symmetry. This completely different temperature dependence of vacancies and interstitials is not dominated by their energy, which is almost degenerated, but by the entropy. The entropic and energetic contributions can be accurately determined from a second-order expansion of the energy with respect to displacements.

The kinetics of the defects is well described by a master equation in a multistate Markov model. The states are different symmetries of the defects, and the rates between different states are determined from time correlation functions, which we measure in experiment and computer simulations.

This work presents a detailed study on the defect energetics and dynamics of point defects in two-dimensional materials. We hope that this motivates experiments in other two-dimensional systems. A particular future question that becomes accessible, e.g., in ultracold dipolar quantum gases [30] and graphene [31], is the role of quantum fluctuations in defect dynamics.

\section{ACKNOWLEDGMENTS}

Work was supported by the Austrian Science Fund (FWF): P 25454-N27 and the German Research Foundation (DFG), SFB-TR6, project C2 and SFB ViCoM (Grant No. F41).
[1] G. I. Taylor, Proc. R. Soc. A 145, 362 (1934).

[2] D. R. Nelson, Defects and Geometry in Condensed Matter Physics (Cambridge University Press, Cambridge, 2002).

[3] R. E. Kusner, J. A. Mann, J. Kerins, and A. J. Dahm, Phys. Rev. Lett. 73, 3113 (1994).

[4] A. H. Marcus and S. A. Rice, Phys. Rev. Lett. 77, 2577 (1996).

[5] K. Zahn and G. Maret, Phys. Rev. Lett. 85, 3656 (2000).

[6] Y. Han, N. Y. Ha, A. M. Alsayed, and A. G. Yodh, Phys. Rev. E 77, 041406 (2008).

[7] S. Deutschländer, A. M. Puertas, G. Maret, and P. Keim, Phys. Rev. Lett. 113, 127801 (2014)

[8] J. Kosterlitz and D. Thouless, J. Phys. C 6, 1181 (1973); B. I. Halperin and D. R. Nelson, Phys. Rev. Lett. 41, 121 (1978); A. P. Young, Phys. Rev. B 19, 1855 (1979); K. J. Strandburg, Rev. Mod. Phys. 60, 161 (1988).

[9] D. Frenkel and J. P. McTague, Phys. Rev. Lett. 42, 1632 (1979).

[10] K. J. Strandburg, S. A. Solla, and G. V. Chester, Phys. Rev. B 28, 2717 (1983).

[11] A. Jaster, Europhys. Lett. 42, 277 (1998).

[12] S. Sengupta, P. Nielaba, and K. Binder, Phys. Rev. E 61, 6294 (2000).

[13] C. H. Mak, Phys. Rev. E 73, 065104 (2006).

[14] H. Shiba, A. Onuki, and T. Araki, Europhys. Lett. 86, 66004 (2009).

[15] E. P. Bernard and W. Krauth, Phys. Rev. Lett. 107, 155704 (2011).

[16] W. Lechner, D. Polster, G. Maret, P. Keim, and C. Dellago, Phys. Rev. E 88, 060402 (2013).
[17] M. Boninsegni, A. B. Kuklov, L. Pollet, N. V. Prokofev, B. V. Svistunov, and M. Troyer, Phys. Rev. Lett. 97, 080401 (2006).

[18] F. Cinti, T. Macrí, W. Lechner, G. Pupillo, and T. Pohl, Nat. Commun. 5, 3235 (2014).

[19] W. Lechner and C. Dellago, Soft Matter 5, 2752 (2009).

[20] W. Lechner and C. Dellago, Soft Matter 5, 646 (2009).

[21] V. Prasad, D. Semwogerere, and E. R. Weeks, J. Phys.: Condens. Matter 19, 113102 (2007).

[22] A. Pertsinidis and X. S. Ling, New J. Phys. 7, 33 (2005).

[23] A. Pertsinidis and X. S. Ling, Phys. Rev. Lett. 87, 098303 (2001).

[24] C. Eisenmann, U. Gasser, P. Keim, G. Maret, and H.-H. von Grünberg, Phys. Rev. Lett. 95, 185502 (2005).

[25] U. Gasser, C. Eisenmann, G. Maret, and P. Keim, ChemPhysChem 11, 963 (2010).

[26] F. Ebert, P. Dillmann, G. Maret, and P. Keim, Rev. Sci. Instrum. 80, 083902 (2009).

[27] S. Jain and D. R. Nelson, Phys. Rev. E 61, 1599 (2000).

[28] A. Libál, C. Reichhardt, and C. J. Olson Reichhardt, Phys. Rev. E 75, 011403 (2007).

[29] S. Fortune, Algorithmica 2, 153 (1987).

[30] H. P. Büchler, E. Demler, M. Lukin, A. Micheli, N. Prokofev, G. Pupillo, and P. Zoller, Phys. Rev. Lett. 98, 060404 (2007); W. Lechner, H.-P. Büchler, and P. Zoller, ibid. 112, 255301 (2014); W. Lechner and P. Zoller, ibid. 111, 185306 (2013).

[31] J. H. Warner, E. R. Margine, M. Mukai, A. W. Robertson, F. Giustino, and A. I. Kirkland, Science 337, 209 (2012). 